\title{
Selectivity of Recording of Neural Signals with Micromachined Intraneural Multi Electrodes
}

\author{
Wim L. C. Rutten, Member IEEE, Tom H. Rozijn and Jan H. Meier \\ Institute of Biomedical Technology, Faculty of Electrical Engineering, University of Twente, PO Box 217, 7500 AE \\ Enschede, the Netherlands
}

\begin{abstract}
The number of afferent fibers in a peripheral nerve fascicle and the electrical volume conduction in neural tissue determine the interelectrode spacing, needed for selective recording with multicontact devices. These factors taking into account, and also assuming uniform distribution of fibers throughout the fascicle cross section, it is calculated that for selective sensing in peroneal nerve fascicles, the electrode spacing must be about $80 \mu \mathrm{m}$. This is for the nerve, embedded in its natural saline environment. The selectivity, thus reached, however reduces drastically to zero when an insulating cuff is positioned around the nerve at the position of the intraneural multi-electrode device.
\end{abstract}

\section{INTRODUCTION}

In case of selective recording of single neve fiber activity, the small diameter of axons (micrometers) asks for miniature multi-contact electrode devices [1, 3]. This paper examines selectivity of recording and calculates the corresponding necessary electrode spacing.

The same type of calculation, as previously made for the case of selective stimulation of nerve fibers in rat peroneal nerve (isotropic conductor, local approach [1]) could be applied to the case where the device is used to sense natural activity of type I afferent fibres (muscle spindle and tendon organs). They constitute about $24 \%$ of the total number of fibers, which is 250 fibers in rat peroneal nerve. These numbers would lead to a calculated electrode interdistance of $113 \mu \mathrm{m}$.

However, this method of calculating an interdistance estimate is too simplified for recording purposes. This is due to the fact that an active fiber cannot be modeled as a local one-node-of-Ranvier point source (one active node), as in an active fiber about twenty active nodes contribute to a travelling action potential, i.e. twenty nodes inject current to the nerve tissue. Therefore, the following presents a more accurate procedure.

\section{SELECTIVITY OF RECORDING}

When two (not overlapping) action potential trains are sensed by one electrode, the trains can be detected separately when the selectivity ratio $S$ of their amplitudes $V_{1}$ and $V_{2}$ exceeds a certain threshold, i.e. when $S \geq S_{\text {th }}$, for example $S \geq 1.1$, or $S \geq 2$.

Quantitative insight in the selectivity ratio $S$ as a function of spatial and conductivity parameters may be obtained by the combined use of an electrode lead field model and by a probability model .

\section{A. Lead field model}

Figurc 1 shows the lead field for a monopolar electrode. The horizontal axis is the axial direction of the nerve. The lead field indicates for every point in the fascicle how strong a fiber is 'seen' at the electrode when a node of the fiber is positioned at that point. Figure 1 shows the case for realistic fibers in which 20 active nodes shape the action potential (see [3] ). The internode distance is $1 \mathrm{~mm}$ (corresponding to a fiber with $10 \mu \mathrm{m}$ diameter), the fascicle diameter is 0.5 $\mathrm{mm}$. Figure 1 shows one elementary section of a periodical function (periodicity is $1 \mathrm{~mm}$, i.e. the internode distance). See [2] and [3] for the volume conduction model of the nerve. Conductivity parameters are 0.1 (radial conductivity), 0.5 (axial), 0.1 (epineural conductivity), 0.1 (extraneural conductivity), all in $\Omega / \mathrm{m}$, and $2000\left(1 / \Omega \mathrm{m}^{2}\right.$,perineural sheath conductivity). Radii are $250 \mu \mathrm{m}$ (fascicle) and $270 \mu$ $\mathrm{m}$ (nerve). Propagation velocity was $57 \mathrm{~m} / \mathrm{s}$.

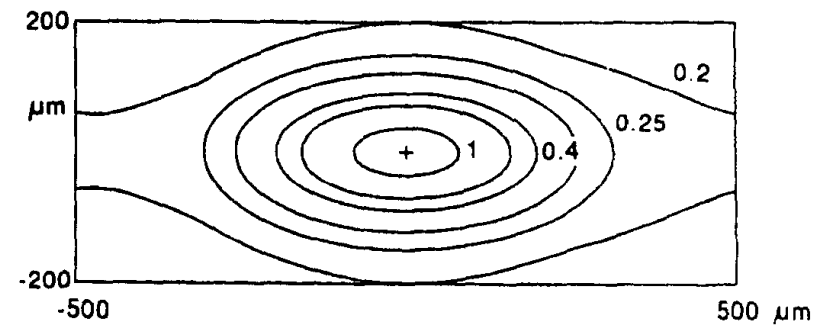

Fig. 1. Lead field equipotential comtours in a crossectional plane through the axis of the nerve (axial direction is horizontally and radial direction is vertically). The electrode is indicated by the + symbol. The active fibers have twenty active nodes shaping the action potential.

Figure 1 indicates, for example, that the selectivity ratio $S$ is two for one node of one fiber at the innermost contour and another node - of a second fiber- at the next contour, which is radially $33 \mu \mathrm{m}$ away. It shows that with a lower discrimation limit of $\mathrm{S}=2$ the two fibers must be separated more than $33 \mu \mathrm{m}$ radially (with the first fiber at the central contour). Conversely, two electrodes separated by a radial distance of $33 \mu \mathrm{m}$, at the most central and the second 
contour. would measure the signal coming from one central fiber. with an amplitude ratio of two.

\section{B. Probabilitu medel}

The next step is the calculation of the probability $P$ that $S$ cquals or excecds a certain threshold (c.g 1.1, 1.5 or 2) for an elcctrode at an arbitrary place in the bundle of nerve fibers. The calculations were done in a 'Monte Carlo' way. One electrode was placed at the center of the nerve. Fourty or 250 fibers werc distributed randomly over the nerve cross section. with random node position (but fixed internode distance along each fiber). This was repeated to yicld 5000 realisations. For even realisation the ratio $S$ of the two strongest action potentials (usually those nearest to the electrode). was calculated. using the lead ficlds. The probability $P\left(S>S_{1 h}\right)$ is the fraction of rcalisations for which $S>S_{t h}$

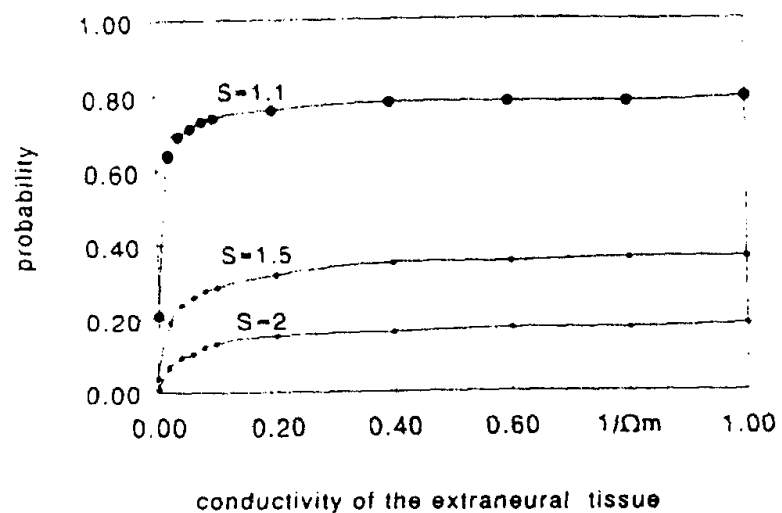

Fig. 2. The probability (density)that the measured action potentials from those wo fibers. whith are nearest to is central monopolar electrode have an amplitude ratio $S-1.1$. 1.5 or 2 . as a function of the conductivity of the extrancural ussue. The nerve has $\$ 0$ active fibers ( 20 attive nodes each)

Figure 2 shows this probability as a function of the extraneural conductivity (electrode in the center of the nerve). for selectivity thresholds $S=1.1,1.5$ and 2. with to active fibers. A dramatic decrease in the ability to discriminate two trains is seen when the nerve is insulated from its surrounding tissue. i.e. for zero extraneural conductivity. This may be the case when a lightly fitting insulating (e.g. Silastic) cuff is used around the nerve at the position of the intraneural device. like in some prototypes of recording devices [5], [6].

The curves saturate to the respective limiting values $\mathrm{S}^{-3}$. which is the value that is to be expected in an analytical Poisson-model calculation (infinite space. isotropic conductivity. $(1 .+1)$.

From Monte Carlo simulations with 250 afferent fibers it can be derived that a central electrode has a volume with a radius of $40 \mu \mathrm{m}$ around it. inside which the probability is 0.99 that the fiber with the targest signal at the clcctrode lies inthat volume. such that the amplitude ratio to the second largest signal (lving anywhere) $S \geq 2$. For $S \geq 1.1$ this radius is $75 \mu \mathrm{m}$.

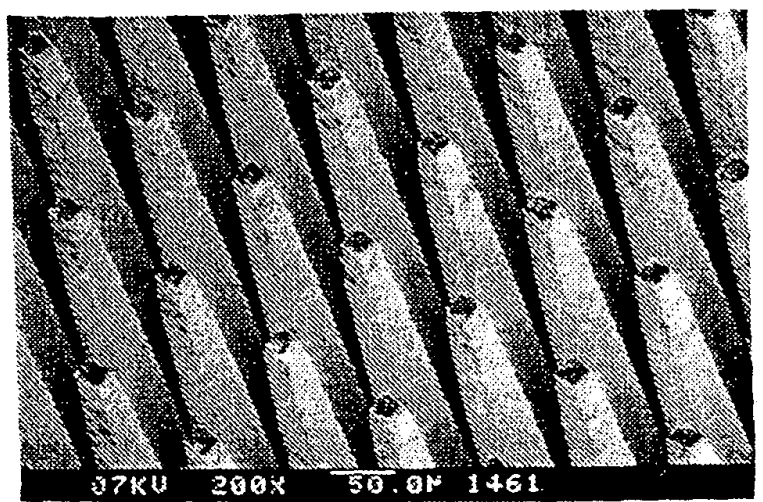

Fig. 3. Basic structure of tapeted silicon 'needle' devike for a 3-dimensional multicontact neural interface. Distance between electrode sites at the tip is 100 mictometer. [3].

\section{CONCLL'SIONS}

These outcomes indicate that mului clectrode devices should have inter electrode distances of $80 \mu \mathrm{m}$ and $150 \mu \mathrm{m}$ ( $\mathrm{S} \geq 2$ and $\geq 1$. $\mathrm{h}$. respectively). like in fig. 3 . and that the use of insulating cuffs around the nerve should be avoided. However. it should be noted that the calculations assume a uniform distribution of fibers over the crossection of the fascicle, which may not be true in reality. Especially the organization of peripheral axons in functional clusters in a localized region of the cross section of the nerve fascicle may drastically modify the demands for selective recording. i.e. lcad to electrode designs with much less electrode spacing.

\section{REFERE.VCES}

11] W.L.C. Rutter H. van W'ier and J.H.MI. Put " Sensitivity and selectivity of intraneural stimulation using a silicon electrode amay." IFf.t. Transatussis Biomedical Fngineering 38(2), 1991, 192-198

(2) J.H. Meier, W.I.C. Rutten . A.E. Zoutman. H.B.K. Boom and P. Bergveld. "Simulation of multipolar fibre selative neural stimulation using intrafascicular electrodes." IEEE Transactions Biomedical Engineering 39/2) 122-1.34. 1992

[3] J.H. Meicr. "Selectivity and design of neuro-electronic intirfaces." Thesis. Lniversity of Twente. Enschede. the Vetherlands. 1992. ISBN Y()9004835-9

[4] I.D. Clark and D.J. Fdell. "Flectrode spacing considerations for neural signal transducers," Proc. 9th .Annual Conf. of the IEFE.E.MBS Socierv. PP 490-491. 1987.

[5] D.J.F.dell. "A peripheral nen'e indormation transducer lin amputees. long-term multichannel recordings from rabbit peripheral nenies". IFF.F Transactions Biomedical Engineering 33(2). 1986. 203-214.

[6] G.T.A Kovacs, C.W. Stoment and J.M. Rosen. "Kegentration microelectrode array for peripheral nerve recording and stimulatuon". IELL Transactions Biomedical Engineering 39(9), 1992, 893-(\%)2. 